\title{
HUGE INSTABILITIES DRIVEN BY QUANTUM FLUCTUATIONS
}

\author{
V. A. Zagrebnov \\ Centre de Physique Théorique and Département de Physique, Université de la Méditerranée (Aix-Marseille II), \\ Luminy-Case 907, F-13288 Marseille, Cedex 09, France; E-mail: zagrebnov@cpt.univ-mrs.fr
}

(Received September 26, 1996)

\author{
Quantum Fluctuations Approach is proposed as a key to understand instabilities in a Quantum \\ Ferroelectric Model. \\ Key words: quantum fluctuations, instabilities, phase transition. \\ PACS number(s): 05.90.+m
}

Dedicated to Ivan Vakarchuk in occasion of his 50th anniversary

\section{INTRODUCTION AND SETUP OF THE PROBLEM}

\section{A. Mathematical Framework}

To describe any ( $\mathbb{Z}^{d}$-lattice) quantum statistical model, one has to start from microscopical dynamical system which is a triplet $\left(\mathcal{A}, \omega, \alpha_{t}\right)$ where:

(a) $\mathcal{A}=\cup_{\Lambda} \mathcal{A}_{\Lambda}$ is the quasi-local algebra of observables, here $\Lambda$ are bounded subset of $\mathbb{Z}^{d}$ and $\left[\mathcal{A}_{\Lambda^{\prime}}, \mathcal{A}_{\Lambda^{\prime \prime}}\right]=$ 0 if $\Lambda^{\prime} \cap \Lambda^{\prime \prime}=\emptyset$.

(b) $\omega$ is a state of $\mathcal{A}$. Let $\tau_{x}$ be space translation automorphism of translations over the distance $x \in \mathbb{Z}^{d}$, i.e., $\tau_{x}: A \in \mathcal{A}_{\Lambda} \rightarrow \tau_{x}(A) \in \mathcal{A}_{\Lambda+x}$. Then the state $\omega$ is translation invariant if $\left(\omega \circ \tau_{x}(A) \equiv \omega\left(\tau_{x}(A)\right)=\omega(A)\right.$ and space clustering if $\lim _{|x| \rightarrow \infty} \omega\left(A \tau_{x}(B)\right)=w(A) \omega(B)$ for $A, B \in \mathcal{A}$.

(c) $\alpha_{t}$ is dynamics described by the family of local Hamiltonians $\left\{\mathcal{A}_{\Lambda}\right\}_{\Lambda}$. Usually, $\alpha_{t}$ is defined as a norm limit of the local dynamics: $\alpha_{t}(A)=$ $\lim _{\Lambda} \exp \left(i t\left[\mathcal{A}_{\Lambda}\right.\right.$, o] $)(A) \equiv \lim _{\Lambda} \exp \left(i t \mathcal{A}_{\Lambda}\right) A \exp \left(-i t \mathcal{A}_{\Lambda}\right)$, i.e., $\alpha_{t}: \mathcal{A} \rightarrow \overline{\mathcal{A}}$-norm-closure of $\mathcal{A}$. For equilibrium states one assumes that $\omega \circ \alpha_{t}=\omega$ (time invariance).

Usually one assumes also that the space and time translations commute: $\tau_{x}\left(\alpha_{t}(\mathrm{o})\right)=\alpha_{t}\left(\tau_{x}((\circ))\right.$.

On the way from the micro system $\left(\mathcal{A}, \omega, \alpha_{t}\right)$ to macro system of physical observables, one has to distinguish two essential classes.

The first one (macro I) correponds to the Weak Law of Large Numbers (WLLN) and well-suited for description of order parameters in the system. Formally this class of observables is defined as follows (WLLN):

For any $A \in \mathcal{A}$ the local space mean mapping $m_{\Lambda}: A \rightarrow$ $m_{\Lambda}(A) \equiv|\Lambda|^{-1} \sum_{x \in \Lambda} \tau_{x}(A)$, Then, the limiting mapping $m: A \rightarrow \mathcal{C}$ exist in the weak operator topology induced by the state $\omega$ :

$$
m(A)=w-\lim _{\Lambda} m_{\Lambda}(A) .
$$

Let $m(\mathcal{A})=\{m(A): A \in \mathcal{A}\}$. Then the macro system $\mathrm{I}$ has the following properties:

(Ia) $m(\mathcal{A})$ is a set of observables at infinity because $[m(\mathcal{A}), \mathcal{A}]=0$.

(Ib) $m(\mathcal{A})$ is an abelian algebra and $m(A)=$ $\omega(A) . \mathbf{1}$, hence the states on $m(\mathcal{A})$ are probability measures.

(Ic) the map $m: \mathcal{A} \rightarrow m(\mathcal{A})$ is not injective, e.g., $m\left(\tau_{a}(A)\right)=m(A)$. this is the mathematical of the coarse graining under the WLLN.

(Id) The macro-dynamics $\tilde{\alpha}_{t} m(A) \equiv m\left(\alpha_{t} A\right)$ induced by the microdynamics (c) on $m(\mathcal{A})$ is trivial: $m\left(\alpha_{t} A\right)=\omega\left(\alpha_{t}(A)\right) . \mathbf{1}=\omega(A) . \mathbf{1}=m(A)$.

The second class of macro-observable (macro II) corresponds to the Quantum Central Limit (QCL), which is well-suited for description of (quantum) fluctuations and, in particular, for description of collective and elementary exitations (phonons, plasmons, etc) in many body quantum systems, see e.g. $[1,2]$.

Let $A \in \mathcal{A}_{s a}=\left\{B \in \mathcal{A}: B=B^{*}\right\}$. Then one can define the local mapping $F_{k, \Lambda}^{\delta}: A \rightarrow F_{k, \Lambda}^{\delta}(A)$ where

$$
\begin{aligned}
& F_{k, \Lambda}^{\delta_{A}}(A)=\frac{1}{|\Lambda|^{\frac{1}{2}+\delta_{A}}} \sum_{x \in \Lambda}\left(\tau_{x}(A)-\omega(A)\right) e^{i k x} \\
& k, \delta_{A} \in \mathbb{R}^{1}
\end{aligned}
$$

which is nothing but the local fluctuation operator for the mode $\mathrm{k}$. If $\delta=0$, this fluctuation operator is called normal. The next important concept is due to [3-6]:

Central Limit Theorem Let

$$
\gamma_{\omega}(r)=\sup _{\Lambda, \Lambda^{\prime}} \sup _{\substack{A \in \mathcal{A}_{\Lambda} \\ B \in \mathcal{A}_{\Lambda^{\prime}}}}\left\{\frac{\omega(A B)-\omega(A) \omega(B)}{\|A\| \cdot\|B\|}: r \leq \operatorname{dist}\left(\Lambda, \Lambda^{\prime}\right)\right\}
$$

and 


\section{A. ZAGREBNOV}

$$
\sum_{x \in \mathbb{Z}^{d}} \gamma_{\omega}(|x|)<\infty
$$

Then, for any $A \in \mathcal{A}_{s a}$, the corresponding limiting characteristic fonction exists for the normal fluctuation operator (i.e. for $\delta_{A}=0$ ) for the mode $k=0$ :

$$
\lim _{\Lambda} \omega\left(e^{i u F_{\Lambda}(A)}\right)=e^{-\frac{u^{2}}{2} S_{\omega}(A, A)}, u \in \mathbb{R}^{1}
$$

where $S_{\omega}(A, B)=\operatorname{Re} \sum_{x \in \boldsymbol{Z}^{d}} \omega\left((A-\omega(A)) \cdot \tau_{x}(B-\omega(B))\right)$.

(II a) The result (1.3) establishes the meaning of the Quantum Central Limit. If (1.3) exists for $\delta_{A} \neq 0$ with modified bilinear form

$$
S_{\omega, \delta_{A, B}}(A, B)=\lim _{\Lambda} R e \frac{1}{|\Lambda|^{\delta_{A}+\delta_{B}}} \sum_{x \in \boldsymbol{Z}^{d}} \omega\left((A-\omega(A)) \cdot \tau_{x}(B-\omega(B))\right)
$$

we say about existence of QCL for abnormal fluctuations for $k=0$

$$
\lim _{\Lambda} F_{\Lambda}^{\delta_{A}}(A)=F^{\delta_{A}}(A)
$$

(II b) Let be $\mathcal{A}_{\text {as }}$ consedered now as a vectorspace with the symplectic form $\sigma_{\omega}(\mathrm{o}, \circ)$ correctly defined by the WLLN for the case $\delta_{A}+\delta_{B}=0$

$$
i \sigma_{\omega}(A, B) . \mathbf{1}=\lim _{\Lambda}\left[F_{\Lambda}^{\delta_{A}}(A), F_{\Lambda}^{\delta_{B}}(B)\right]=2 i \operatorname{Im} \sum_{y \in \mathbb{Z}^{d}}\left(\omega\left(A \tau_{y} B\right)-\omega(A) \omega(B)\right)
$$

Consider the Weyl algebra $W\left(\mathcal{A}_{s a}, \sigma_{\omega}\right)$, i.e., Weyl operators $W: A \in \mathcal{A}_{s a} \rightarrow W(A)$ such that

$$
W(A) W(B)=W(A+B) e^{-\frac{i}{2} \sigma_{\omega}(A, B)}
$$

where $A, B \in \mathcal{A}_{s a}$, acting on some Hilbert space.

Reconstruction Theorem: Let $\tilde{\omega}$ be quasi-free state on $W\left(\mathcal{A}_{s a}, \sigma_{\omega}\right)$ defined by the form $S_{\omega}(\circ, \circ)$

$$
\tilde{\omega}(W(A))=e^{-\frac{1}{2} S_{\omega}(A, A)}
$$

as far as $W(A)=e^{i \Phi(A)}$, where $\Phi: A \rightarrow \Phi(A)$ are boson field operators acting on the representation space $\mathcal{H}_{\tilde{\omega}}$ of the state $\tilde{\omega}$, the (1.2) and (1.6) give the identification $\mathcal{H}=\mathcal{H}_{\tilde{\omega}}$ and

$$
\lim _{\Lambda} F_{\Lambda}^{\delta_{A}}(A) \equiv F^{\delta_{A}}(A)=\Phi(A)
$$

(II c) The Reconstruction Theorem gives a transition from the micro-system $\left(\mathcal{A}_{s a}, \omega\right)$ to the macrosystem of the fluctuation operators $\left(F\left(\mathcal{A}_{s a}, \sigma_{\omega}\right), \tilde{\omega}\right)$, where $F\left(\mathcal{A}_{s a}, \sigma_{\omega}\right)=\left\{F^{\delta_{A}}(A)_{A \in \mathcal{H}_{s a}}\right\}$ is the $C C R$-algebra on the symplectic space $\left(\mathcal{A}_{s a}, \sigma_{\omega}\right)$, see (1.6) and (1.8).

(II d) The map $F: \mathcal{A}_{s a} \rightarrow F\left(\mathcal{A}_{s a}, \sigma_{\omega}\right)$ is not injective (zero mode coase graining), e.g., $\tilde{\tau}_{x} F(A) \equiv$
$F\left(\tau_{x}(A)\right)=F(A)$, it has (non-trivial) macro-dynamics $\tilde{\alpha}_{t}(F(A)) \equiv F\left(\alpha_{t}(A)\right)$. Therefore, the macro-system II defined by the algebra of fluctuation operators is the triplet $\left(F\left(\mathcal{A}_{s a}, \sigma_{\omega}\right), \tilde{\omega}, \tilde{\alpha}_{t}\right)$.

\section{B. Fluctuation Operators}

Identification of the algebra of the fluctuation operators $F\left(\mathcal{A}_{s a}, \sigma_{\omega}\right)$ for a given micro-system $\left(\mathcal{A}, \omega, \alpha_{t}\right)$ with the CCR-algebra of the boson field operators supplies a mathematical description of so-called collective excitations (phonons, plasmons, etc $[1,2]$ ) in the pure state $\omega$. The same approach gives as well a break into the mathematical foundation of another physical concept: the Linear Response Theory [6].

In the latter case, it became clear that algebra of fluctuations is more sensible with respect to "gentle" pertubations of the microscopic Hamiltonian than, e.g., algebra at infinity $m(\mathcal{A})$. This property gets even more sound if the equilibrium state $\omega$ (being pure) belongs to the critical domain [7-10]. In this case, pertubations of microscopic Hamiltonien which do not change equilibrium state $\omega$ ("gentle" pertubations) can produce different algebras of fluctuations independent of quantum or classical nature of the micro-system.

The idea of pertubation of Hamiltonien to produce pure equilibrium states comes back to Bogoliubov quasi- 
averages [11], generalized later to include mixed states [12]. It can be formulated as follows

(i) Let $\left\{B_{l}=\tau_{l}(B)\right\}_{l \in \mathbb{Z}^{d}}$ be operators breaking the symmetry of the initial system

$$
H_{\Lambda}(h) \equiv H_{\Lambda}-\sum_{l \in \Lambda} h_{l} B_{l}, \quad h_{l} \in \mathbb{R}^{1}
$$

(ii) Then the limiting states for $h_{l}=h$

$$
\langle-\rangle=\lim _{h \rightarrow 0} \lim _{\Lambda}\langle-\rangle_{\Lambda, h}
$$

pick out pure states with respect to decomposition corresponding the symmetry (Bogoliubov's quasi-averages).

(ii) If the external field $h=\hat{h} /|\Lambda|^{\alpha}$, then the obvious generalization of (1.10) either $\left(\alpha<\alpha_{c}\right)$ coincides with pure states or give a family of mixed states enumerated by $\hat{h}$ and $\alpha \geq \alpha_{c}$, see [7, 12]. For an interesting application of this concept in the nontranslation-invariant case $h_{l}=\hat{h}_{l} /|\Lambda|^{\alpha}$ see [13].

As it was discovered in [7], the algebra of fluctuations for a quantum model of ferroelectric (structural phase transitions) depends on the parameter $\alpha$ in the critical domain (below the critical line) even for the pures states, i.e., for $\alpha<\alpha_{c}=1$ one gets $\delta_{Q}=\alpha / 2$, while $\delta_{P}=0$ (for $T \neq 0, T$ is the temperature), see (1.3), (1.6). Here $A=Q$ and $B=P$ are respectively the atomic displacement and momentum operators in the site $(l=0)$ of $\mathbb{Z}^{d}$. The second observation made in [7] concernes the quantum nature of the critical fluctuations, i.e., fluctuations in the pure state $\omega$ which belongs to the critical line. It was shown that expected abelian properties of critical fluctuations can changes into non-abelian commutations between $F^{\delta_{Q}}(Q)$ and $F^{\delta_{P}}(P)$ with $\delta_{Q}=-\delta_{P}>0$, see (1.6), for $T=0, \lambda$. Here, $\lambda=\hbar / \sqrt{m}$ is quantum parameter of the model where $\mathrm{m}$ is the mass of atoms in the nodes of lattice $\mathbb{Z}^{d}$.

The aim of the present note is to study the algebra of fluctuation operators and its instability driven by quantum fluctuations on the critical line. As far as corresponding critical equilibrium state use to have long-range correlations, the critical fluctuations are unticipated to be sensitive with respect to above "gentle" pertubations $h=\hat{h} /|\Lambda|^{\alpha}$. On the other hand, they have to be sensitive to decay of a direct interaction between particles: in our model, the decay of the harmonic force matrix elements is given by

$$
\phi_{l, l^{\prime}} \sim\left|l-l^{\prime}\right|^{-(d+\sigma)} \text { for }\left|l-l^{\prime}\right| \longrightarrow \infty .
$$

If $\sigma \geq 2$ one classified (1.11) as a short range while the case $\overline{0}<\sigma<2$ is classified as long range because the corresponding lattice Fourier-transform has the following asymptotics for $k \rightarrow 0$

$$
\tilde{\phi}(k) \sim\left\{\begin{array}{ll}
a^{\sigma} k^{\sigma}+o\left(k^{\sigma}\right), & 0<\sigma<2 \\
a^{2} k^{2}+o\left(k^{2}\right), & \sigma \geq 2
\end{array} .\right.
$$

Therefore, our purpose is to find exponents $\delta_{A}$ as the function of the parameter $\alpha$ and $\sigma$ for the quantum ferroelectric model [7]. Note that $\delta_{A}=\delta_{A}(\alpha, \sigma)$ is directly related to the critical exponent $\eta$ describing decay of the two-point correlation function on the critical line: $\eta=2-2 d \delta_{A}[14]$.

The paper is organized as follows. In the next section, we recall our model and describe its thermodynamics related to fluctuations on the critical line. Detailed analysis of the latter as well as the main result: calculation of $\delta_{Q}(\alpha, \sigma)$ and $\delta_{P}(\alpha, \sigma)$ are collected in section 3. Concluding remarks are postponed up to section 4 .

\section{MODEL AND PHASE TRANSITION}

\section{A. The Ferroelectric Model}

Let $\mathbb{Z}^{d}$ the d-dimensional square lattice. At each lattice site $l$ occupied by a particle with mass $\mathrm{m}$, we associate the position operator $Q_{l} \in \mathbb{R}^{1}$ and the momentum operator $P_{l}=(\hbar / i)\left(\partial / \partial Q_{l}\right)$. Let $\Lambda$ be a finite subset of $\mathbb{Z}^{d}, V=|\Lambda|$ and $\Lambda^{*}$ is the dual. The local Hamiltonian of our model is given by

$$
\begin{aligned}
& H_{\Lambda}=\sum_{l \in \Lambda} \frac{P_{l}^{2}}{2 m}+\frac{1}{4} \sum_{l, l^{\prime}} \phi_{l, l^{\prime}}\left(Q_{l}-Q_{l^{\prime}}\right)^{2} \\
& +\sum_{l \in \Lambda} U\left(Q_{l}\right)-h \sum_{l \in \Lambda} Q_{l} .
\end{aligned}
$$

The second term of (2.1) represents the harmonic potential between particles, the last term represents the action of an external field and the third one is the anharmonic potential acting on each site $l$. $U$ must have a double potential form to describe a displacive structural phase transition attribuated to one-component ferroelectric. For instance, $U(x)=\frac{a}{2} Q_{l}^{2}+W\left(Q_{l}^{2}\right), a<0$, with $W(x)=\frac{1}{2} b x^{2}, b>0$ or $a>0$ with $W(x)=$ $\frac{1}{2} b \exp (-\eta x), \eta>0$ and $b>0$ sufficiently large to destabilize the a-term. In that way, (2.1) becomes

$$
\begin{aligned}
& H_{\Lambda}=\sum_{l \in \Lambda} \frac{P_{l}^{2}}{2 m}+\frac{1}{4} \sum_{l, l^{\prime}} \phi_{l, l^{\prime}}\left(Q_{l}-Q_{l^{\prime}}\right)^{2}+\frac{a}{2} \sum_{l \in \Lambda} Q_{l}^{2} \\
& +\sum_{l \in \Lambda} W\left(Q_{l}^{2}\right)-h \sum_{l \in \Lambda} Q_{l} .
\end{aligned}
$$

The model (2.2) can be solved exactly if we applie the following rule (spherical approximation) known as the concept of Self-Consistent Phonons, see [15, 16].

$$
\sum_{l \in \Lambda} W\left(Q_{l}^{2}\right) \longrightarrow V W\left(\frac{1}{V} \sum_{l \in \Lambda} Q_{l}^{2}\right)
$$

Then we get Hamiltonian of our model 


\section{A. ZAGREBNOV}

$$
H_{\Lambda}=\sum_{l \in \Lambda} \frac{P_{l}^{2}}{2 m}+\frac{1}{4} \sum_{l, l^{\prime}} \phi_{l, l^{\prime}}\left(Q_{l}-Q_{l^{\prime}}\right)^{2}+\frac{a}{2} \sum_{l \in \Lambda} Q_{l}^{2}+V W\left(\frac{1}{V} \sum_{l \in H_{\Lambda}} Q_{l}^{2}\right)-h \sum_{l \in \Lambda} Q_{l} .
$$

The model (2.3) can be solved by the Approximating Hamiltonian Method [17]. We obtain the free energy density (see, e.g., appendix of [7])

$$
\begin{aligned}
f_{\Lambda}\left[H_{\Lambda}\left(c_{\Lambda, h}(T, \lambda)\right)\right] & =\frac{1}{\beta V} \sum_{q \in \Lambda^{*}} \ln \left[2 \sinh \frac{\beta \lambda \Omega_{q}\left(c_{\Lambda, h}(T, \lambda)\right)}{2}\right]-\frac{1}{2} \frac{h^{2}}{\Delta\left(c_{\Lambda, h}\left(T_{c}(\lambda), \lambda\right)\right)} \\
& +\left[W\left(c_{\Lambda, h}(T, \lambda)\right)-c_{\Lambda, h}(T, \lambda) W^{\prime}\left(c_{\Lambda, h}(T, \lambda)\right)\right]
\end{aligned}
$$

where $c_{\Lambda, h}(T, \lambda)$ is the solution of the self-consistency equation

$$
c_{\Lambda, h}(T, \lambda)=\frac{h^{2}}{\Delta^{2}\left(c_{\Lambda, h}(T, \lambda)\right)}+\frac{1}{V} \sum_{q \in \Lambda^{*}} \frac{\lambda}{2 \Omega_{q}\left(c_{\Lambda, h}(T, \lambda)\right)} \operatorname{coth} \frac{\beta \lambda}{2} \Omega_{q}\left(c_{\Lambda, h}(T, \lambda)\right)
$$

with

$$
\begin{aligned}
& \Omega_{q}^{2}\left(c_{\Lambda, h}(T, \lambda)\right)=\Delta\left(c_{\Lambda, h}(T, \lambda)\right)+\omega_{q}^{2}, \\
& \Delta\left(c_{\Lambda, h}(T, \lambda)\right)=a+2 W^{\prime}\left(c_{\Lambda, h}(T, \lambda)\right), \\
& \omega_{q}^{2}=\tilde{\phi}(0)-\tilde{\phi}(q), \\
& \tilde{\phi}(q)=\sum_{l \in \Lambda} \phi_{l, 0} \exp (-i q l), \\
& \lambda=\frac{\hbar}{\sqrt{m}} \\
& \beta=(k T)^{-1} .
\end{aligned}
$$

Here, $\left\{\Omega_{q}\right\}_{q \in \Lambda^{*}}$ are frequencies of the approximating harmonic Hamiltonian [7] and $\Delta\left(c_{\Lambda, h}\left(T_{c}(\lambda), \lambda\right)\right)$ is a gap in the spectrum of (self-consistent) phonons.

Finally, $\lambda$ is the quantum parameter of the model.

The approximating Hamiltonian method gives us the condition of stability of our model

$$
\Delta(c(T, h))=\lim _{\Lambda} \Delta\left(c_{\Lambda, h}\left(T_{c}(\lambda), \lambda\right)\right) \geq 0 .
$$

Let $a>0$ and $W: \mathbb{R}_{+}^{1} \rightarrow \mathbb{R}_{+}^{1}$ be a monotonous decreasing function with $W^{\prime \prime}(c) \geq w>0$. Then by $(2.7)$ and (2.12) one gets for the stability domain: $D=\left[c^{*}, \infty\right)$ such that

$$
c^{*}=\inf \{c ; c \geq 0, \Delta(c) \geq 0\}
$$

i.e., $\Delta\left(c^{*}\right)=0$.

\section{B. The Phase Transition}

To study the phase diagram of the model (2.3) we have to pass to equation (2.5) in the thermodynamic limit $\Lambda \longrightarrow \mathbb{Z}^{d}$ :

$$
c(T, h)=\rho(c(T, h), T, \lambda)+I_{d}(c(T, h), T, \lambda)
$$

where

$$
\rho(T, \lambda)=\lim _{\Lambda}\left\{\frac{1}{V} \frac{\lambda}{2 \sqrt{\Delta\left(c_{\Lambda, h}\left(T_{c}(\lambda), \lambda\right)\right)}} \operatorname{coth} \frac{\beta \lambda}{2} \sqrt{\Delta\left(c_{\Lambda, h}\left(T_{c}(\lambda), \lambda\right)\right)}+\frac{h^{2}}{\Delta^{2}\left(c_{\Lambda, h}(T, \lambda)\right)}\right\}
$$

and

$$
I_{d}(c(T, h), T, \lambda)=\frac{\lambda}{(2 \pi)^{d}} \int_{q \in B_{d}} d^{d} q \frac{1}{2 \Omega_{q}} \operatorname{coth} \frac{\beta \lambda}{2} \Omega_{q}(c(T, h)) .
$$




\section{HUGE INSTABILITIES DRIVEN BY QUANTUM FLUCTUATIONS}

Here, $B_{d}=\left\{q \in \mathbb{R}^{d} ;|q| \leq \pi\right\}$ is the first Brillouin zone.

Let consider two cases: (a) $h=0$ and (b) $h \neq 0$.

(a) $h=0$ : From (2.14),(2.16), one easily gets that for $T=0$, there is $\lambda_{c}$ such that $c^{*} \leq I_{d}\left(c^{*}, 0, \lambda\right)$ for $\lambda \geq \lambda_{c}$. Then line of critical temperatures $T_{c}=T_{c}(\lambda)$ which separates the phase diagram is defined by the condition

$$
c^{*}=I_{d}\left(c^{*}, T, \lambda\right), \lambda \leq \lambda_{c} .
$$

So we obtain the two domains:

- $\rho(c(T, h), T, \lambda)=0$,

- $\rho(c(T, h), T, \lambda)>0$.

For $\lambda<\lambda_{c}$ fixed, by looking along the horizontal ( $\lambda=$ const $)$ line, we observe the well-known temperaturedriven phase transition at $T_{c}(\lambda)>0$ with an order parameter related to $\rho$. Then, for $T<T_{c}(0)$ fixed, by looking along the vertical ( $T=$ const) line, the same occurs. Here, it is the quantum-parameter driven phase transition.

Note that for $\lambda>\lambda_{c}$, i.e. for light atoms, the temperature-driven phase transition is suppressed by quatum tunneling or quatum fluctuations. Decreasing of $T_{c}(\lambda)$ for light atoms is well-known as isotopic effect in ferroelectrics [18]. For the proof that one has the same effect in the model (2.3) including the existence of $\lambda_{c}$, see [7].The proof for the original model (2.1) has been obtained recently in [19].

At last, because of the $\mathbb{Z}^{2}$ symmetry of the Hamiltonian (2.3): $Q_{l} \rightarrow-Q_{l}$, on has

$$
\eta\left(Q_{l}\right) \equiv \lim _{\Lambda} \eta_{\Lambda}\left(Q_{l}\right) \equiv \lim _{\Lambda}\left\langle Q_{l}\right\rangle_{H_{\Lambda}(h=0)}=0
$$

(b) $h \neq 0$ :

Then we obtain

$$
\eta\left(Q_{l}\right)=\frac{h}{\Delta\left(c_{h}\right)}
$$

For disordered phase, we have $\lim _{h \rightarrow 0} c_{h}(T, \lambda)=$ $c(T, \lambda)>c^{*}$. So $\Delta(c)>0$ and

$$
\lim _{h \rightarrow 0} \eta\left(Q_{l}\right)=0
$$

For ordered phase, we have $\lim _{h \rightarrow 0} c_{h}(T, \lambda)=c^{*}$, then

$$
\rho\left(c^{*}, T, \lambda\right)=c^{*}-I_{d}\left(c^{*}, T, \lambda\right)=\lim _{h \rightarrow 0} \frac{h^{2}}{\Delta^{2}\left(c_{h}\right)}>0 .
$$

Finally, (2.19) and (2.21) yield yield the values of the physical order parameters

$$
\eta_{ \pm}\left(Q_{l}\right)=\lim _{h \rightarrow \pm 0} \eta\left(Q_{l}\right)= \pm \sqrt{\rho\left(c^{*}, T, \lambda\right)} \neq 0 .
$$

Therefore, using the Bogoliubov quasi-average (1.10), we obtain two extremal translation invariant equilibrium states $\eta_{+}$and $\eta_{-}$, invariant by translation, such that

$$
\eta_{+}\left(Q_{l}\right)=-\eta_{-}\left(Q_{l}\right)=\left[\rho\left(c^{*}, T, \lambda\right)\right]^{\frac{1}{2}} \neq 0
$$

In this case, one can easily check that positions and momenta have normal fluctuations [7]. So, we pass to generalized quasi-average:

$$
h=\frac{\hat{h}}{V^{\alpha}}, \alpha>0
$$

i.e. we couple $\lim h \rightarrow 0$ to $\lim \Lambda \rightarrow \mathbb{Z}^{d}$. This choice of pertubation of (2.3) is flexible enough to scan between "gentle" - "non-gentle" ones. For example, if $\alpha<1$, then limiting equilibrium states for the phase (II) rest pure: $\lim _{\Lambda}\left\langle Q_{l}\right\rangle_{H_{\Lambda}(\hat{h})}=(\operatorname{sign} \hat{h})\left[\rho\left(c^{*}, T, \lambda\right]^{\frac{1}{2}}\right.$, while for $\alpha \geq 1$ $\eta\left(Q_{l}\right)$ becomes

$$
\eta\left(Q_{l}\right)=a \eta_{+}+(1-a) \eta_{-}
$$

where $a$ is computed in the Proposition 4.2 of [7]. Moreover, changes will emerge for fluctuations operators: it is the mean aim of this note.

\section{Phase Transition and Fluctuations}

Consider now the fluctuation operators of position and momentum given by

$$
F_{\delta_{Q}}(Q)=\lim _{\Lambda} \frac{1}{V^{\frac{1}{2}+\delta_{Q}}} \sum_{i \in \Lambda}\left(Q_{i}-\eta_{\Lambda}\left(Q_{i}\right)\right)
$$

and

$$
F_{\delta_{P}}(P)=\lim _{\Lambda} \frac{1}{V^{\frac{1}{2}+\delta_{P}}} \sum_{i \in \Lambda}\left(P_{i}-\eta_{\Lambda}\left(P_{i}\right)\right)
$$

The calculations of the variances of (2.25) and (2.26) based on the approximating Hamiltonian yield (see e.g. [7])

$$
\lim _{\Lambda} \eta_{\Lambda}\left(\left\{\frac{1}{V^{\frac{1}{2}+\delta_{Q}}} \sum_{i \in \Lambda}\left(Q_{i}-\eta_{\Lambda}\left(Q_{i}\right)\right)\right\}^{2}\right)
$$




\section{A. ZAGREBNOV}

$$
\begin{aligned}
& =\lim _{\Lambda} \frac{1}{V^{2 \delta_{Q}}} \frac{\lambda}{2 \sqrt{\Delta\left(c_{\Lambda, h}\left(T_{c}(\lambda), \lambda\right)\right)}} \operatorname{coth} \frac{\beta \lambda}{2} \sqrt{\Delta\left(c_{\Lambda, h}\left(T_{c}(\lambda), \lambda\right)\right)}, \\
& \lim _{\Lambda} \eta_{\Lambda}\left(\left\{\frac{1}{V^{\frac{1}{2}+\delta_{P}}} \sum_{i \in \Lambda}\left(P_{i}-\eta_{\Lambda}\left(P_{i}\right)\right)\right\}^{2}\right) \\
& =\lim _{\Lambda} \frac{1}{V^{2 \delta_{P}}} \frac{\lambda m \sqrt{\Delta\left(c_{\Lambda, h}\left(T_{c}(\lambda), \lambda\right)\right)}}{2} \operatorname{coth} \frac{\beta \lambda}{2} \sqrt{\Delta\left(c_{\Lambda, h}\left(T_{c}(\lambda), \lambda\right)\right)} .
\end{aligned}
$$

Here $c_{\Lambda, h}(T, \lambda)$ is solution of the self-consistent equation (2.5). The existence of nontrivial variances $(2.27)$ and (2.28) is sufficient for existence of the corresponding quadratic forms in (1.3) [7].

If $(T, \lambda)$ is above the critical line, one has $\lim _{\Lambda} \Delta\left(c_{\Lambda, h}\left(T_{c}(\lambda), \lambda\right)\right)>c^{*}$, consequently $\delta_{Q}=0=\delta_{P}$, i.e. both momentum and displacement fluctuation operators are normal.

If $(T, \lambda)$ belongs to the critical line and $h \equiv 0$, then for $T_{c}>0$, the momentum fluctuation operator is normal while position fluctuation operator is abnormal with the exponent $\delta_{Q}$ depending on the dimension $\mathrm{d}$ and on $\sigma$, the range of the interactions (1.11). At $T_{c}=0$, momentum fluctuation operator is squeezed $\left(\delta_{P}<0\right)$ while position fluctuation operator is abnormal with $\delta_{Q}>0$ such that $\delta_{Q}+\delta_{P}=0$. This leads to a non-abelian algebra of fluctuation operators [7].

Finally,below the critical line, the quasi-averages controled by an external field $h=\hat{h} / V^{\alpha}$ shows for $T>0$ and finite range interactions $(\sigma \geq 2)$ that momentum fluctuation operator is normal while position fluctuation operator is abnormal with the exponent $\delta$ depended on the dimension $\mathrm{d}$ and on $\alpha$ [7]. Of course, this domain is specific because there one has more than one pure phase. That is why the challenge problem is to study whether there such dependence of exponents $\delta_{Q}, \delta_{P}$ on $\alpha$ in the domain where the equilibrium state is unique. from discussion above, one deduces that the critical line is the only place where something non-trivial could realize.

\section{FLUCTUATIONS ON THE CRITICAL LINE}

\section{A. Preliminaries}

Our challenge is to characterize the exponents $\delta_{Q}$ and $\delta_{P}$ on the critical line as function of $d, \sigma$ and if it is the case of $\alpha$. To this end, we shall proceed as follows. On the critical line, one has (2.17). Hence, $\rho\left(c^{*}, T_{c}(\lambda), \lambda=0\right.$, and (2.15) becomes

$$
\lim _{\Lambda}\left\{\frac{1}{V} \frac{\lambda}{2 \sqrt{\Delta\left(c_{\Lambda, h}\left(T_{c}(\lambda), \lambda\right)\right)}} \operatorname{coth} \frac{\beta \lambda}{2} \sqrt{\Delta\left(c_{\Lambda, h}\left(T_{c}(\lambda), \lambda\right)\right)}+\frac{\hat{h}^{2}}{V^{2 \alpha} \Delta^{2}\left(c_{\Lambda, h}(T, \lambda)\right)}\right\}=0 .
$$

Where due to the choice $h=\hat{h} / V^{\alpha}$ one has

$$
\lim _{\Lambda} \Delta\left(c_{\Lambda, h}\left(T_{c}(\lambda), \lambda\right)\right)=0
$$

Now we have to distinguish two cases

(a) $T_{c}(\lambda)>0$, then Eq.(3.5) is equivalent to

$$
\lim _{\Lambda}\left\{\frac{1}{V \Delta\left(c_{\Lambda, h}\left(T_{c}(\lambda), \lambda\right)\right) \beta_{c}(\lambda)}+\frac{\hat{h}^{2}}{V^{2 \alpha} \Delta^{2}\left(c_{\Lambda, h}(T, \lambda)\right)}\right\}=0
$$

(b) $T_{c}(\lambda)=0$, then Eq.(3.5) is equivalent to

$$
\lim _{\Lambda}\left\{\frac{\lambda}{2 V \sqrt{\Delta\left(c_{\Lambda, h}\left(T_{c}(\lambda), \lambda\right)\right)}}+\frac{\hat{h}^{2}}{V^{2 \alpha} \Delta^{2}\left(c_{\Lambda, h}(T, \lambda)\right)}\right\}=0 .
$$




\section{HUGE INSTABILITIES DRIVEN BY QUANTUM FLUCTUATIONS}

Both cases imply that, for $V \rightarrow \infty$, the gap $\Delta$ (3.4) has the asymptotic behaviour $\Delta \simeq V^{-\gamma}$ with $0<\gamma<1$ and $0<\gamma<\alpha$ or $0<\gamma<1$ and $0<\gamma<\alpha$ correspondingly.

It is equation (2.5) which is the key to get these asymptotics. To make it evident, we rewrite (2.5) as

$$
\left(c_{\Lambda}-c^{*}\right)+\left[c^{*}-I_{d}\left(c_{\Lambda}, T_{c}, \lambda\right)\right]+\left[I_{d}\left(c_{\Lambda}, T_{c}, \lambda\right)-\sum_{q \in \Lambda^{*}, q \neq 0} \frac{\lambda}{2 \Omega_{q}\left(c_{\Lambda}\right)} \operatorname{coth} \frac{\beta \lambda \Omega_{q}\left(c_{\Lambda}\right)}{2}\right]=\left(\frac{\hat{h}}{V^{\alpha} \Delta}\right)^{2}+\frac{1}{V} \frac{\lambda}{2 \sqrt{\Delta}} \operatorname{coth}\left(\frac{\beta \lambda \sqrt{\Delta}}{2}\right) .
$$

The asymptotic behaviour of the left-hand side terms of Eq. (3.8) is calculated in $[7,8]$. Thus, we have to combine these calculations with perturbation due to the term in the right hand-side.

\section{B. $T_{c}(\lambda)>0$ : Abelian Fluctuations}

Lemma 3.1 If $(T, \lambda)$ belongs to the critical line $\left(T_{c}(\lambda), \lambda\right)$ with $T_{c}(\lambda)>0$, then the asymptotic volume behaviour of the gap $\Delta\left(c_{\Lambda, h}\left(T_{c}(\lambda), \lambda\right)\right)$ is defined by

$$
\gamma= \begin{cases}\text { if } d>2 \sigma & \text { for } \alpha<\frac{3}{4}=\alpha_{c} \\ \gamma=\frac{2}{3} \alpha & \text { for } \alpha \geq \frac{3}{4} \\ \gamma=\frac{1}{2} & \\ \text { if } d=2 \sigma & \text { for } \alpha<\frac{3}{4}=\alpha_{c} \\ \gamma=\frac{2}{3} \alpha+0 & \text { for } \alpha \geq \frac{3}{4} \\ \gamma=\frac{1}{2}+0 & \\ \text { if } \sigma<d<2 \sigma & \text { for } \alpha<\frac{1}{2}+\frac{\sigma}{2 d}=\alpha_{c} \\ \gamma=2 \alpha \frac{\sigma}{d+\sigma} & \text { for } \alpha \geq \frac{1}{2}+\frac{\sigma}{2 d} \\ \gamma=\frac{\sigma}{d} & \end{cases}
$$

Proof :

As $T_{c}(\lambda)>0$, the right side of Eq.(3.8) becomes has asymptotics (3.6) or

$$
O\left\{\left(\frac{1}{V^{\alpha} \Delta}\right)^{2}+\frac{1}{V \Delta}\right\}
$$

Let us define $\alpha_{c}$ such that $O\left[\left(1 / V^{\alpha_{c}} \Delta\right)^{2}\right] \sim O(1 / V \Delta)$ Then for the asymptotics (3.9) one obviously has

$$
\left(\left(\frac{1}{V^{\alpha_{c} \Delta}}\right)^{2}+\frac{1}{V \Delta}\right)=\frac{1}{V \Delta}
$$

i.e. at $\alpha_{c}$, the gap $\delta$ has the same asymptotic behaviour as for $h=0$. These cases are considered in details in [8]. To make use of this consideration we have to distinguish three regimes of the potentiel decreasing

$$
\text { (a) } \sigma<d<2 \sigma
$$

From [8] it is known that $\gamma=\sigma / d$, i.e. $\Delta \simeq V^{-\frac{\sigma}{d}}$, then
(3.10) yields

$$
\alpha_{c}=\frac{\sigma}{2 d}+\frac{1}{2}
$$

(b) $d=2 \sigma$

In this limiting case one has $\gamma=\frac{1}{2}+0$, so we obtain

$$
\alpha_{c}=\frac{3}{4}
$$

(c) $d>2 \sigma$

The analysis effected in [8] provides $\gamma=\frac{1}{2}$, then

$$
\alpha_{c}=\frac{3}{4}
$$

Consider the case $\alpha>\alpha_{c}$. Then, the decay of $h=\hat{h} / V^{\alpha}$ is too fast in order that asymptotics (3.6) not be sensitive to $\hat{h}$, i.e. the right side of Eq.(3.8) has asymptotics $(1 / V \Delta)$. This means that asymptotics in $(3.8)$ are the same as for $\hat{h}=0$, i.e. we reproduce the results of [8]:

$$
\begin{gathered}
\gamma=\frac{\sigma}{d} \text { if } \sigma<d<2 \sigma \\
\gamma=\frac{1}{2}+0 \text { if } d=2 \sigma \\
\gamma=\frac{1}{2} \text { if } d>2 \sigma
\end{gathered}
$$

Then we twin to $\alpha<\alpha_{c}$ for the three cases (a), (b) and (c) correspondingly. Now the asymptotics of the first term in (3.8) will dominate.
(a) $\sigma<d<2 \sigma$

Then Eq.(3.8) gets the following asymptotics form

$$
O\left[\left(\Delta+\Delta^{\frac{d}{\sigma}-1}+V^{\frac{\sigma}{d}-1}\right) V^{2 \alpha} \Delta^{2}\right]=O(1) .
$$

By to the condition $\sigma<d<2 \sigma$, one gets

$$
O\left(\Delta^{\frac{d}{\sigma}-1} V^{2 \alpha} \Delta^{2}\right)=O(1)
$$




\section{A. ZAGREBNOV}

Hence

$$
\gamma=2 \alpha \frac{\sigma}{d+\sigma}
$$

(b) $d=2 \sigma$

Then the asymptotic form of (3.8) is

$$
O\left[\left(\Delta+\Delta \ln (\Delta)+V^{\frac{1}{2}}\right) V^{2 \alpha} \Delta^{2}\right]=O(1) .
$$

Therefore we obtain

$$
\gamma=\frac{2 \alpha}{3}+0
$$

Where the zero in (4.15) means logarithmic corrections

$$
\text { (c) } d>2 \sigma
$$

Then the asymptotic form of Eq.(3.8) is

$$
O\left[\left(\Delta+V^{\frac{\sigma}{d}-1}\right) V^{2 \alpha} \Delta^{2}\right]=O(1) .
$$

By $d>2 \sigma$, one can check that

$$
\gamma=\frac{2 \alpha}{3}
$$

The lemma is proven

Proposition 3.2 If $(T, \lambda)$ belongs to the critical line $\left(T_{c}(\lambda), \lambda\right)$ with $T_{c}(\lambda)>0$, then the algebra of fluctuation operators is abelian. The momentum fluctuation operator $F_{\delta_{P}}(P)$ is normal $\left(\delta_{P}=0\right)$ while the position fluctuation operator $F_{\delta_{Q}}(Q)$ is abnormal with a critical exponent given by

$$
\delta= \begin{cases}\text { if } d>2 \sigma & \text { for } \alpha<\frac{3}{4}=\alpha_{c} \\ \delta=\frac{1}{3} \alpha & \text { for } \alpha \geq \frac{3}{4} \\ \delta=\frac{1}{4} & \\ \text { if } d=2 \sigma & \text { for } \alpha<\frac{3}{4}=\alpha_{c} \\ \delta=\frac{1}{3} \alpha+0 & \text { for } \alpha \geq \frac{3}{4} \\ \delta=\frac{1}{4}+0 & \\ \text { if } \sigma<d<2 \sigma & \text { si } \alpha<\frac{1}{2}+\frac{\sigma}{2 d}=\alpha_{c} \\ \delta=\alpha \frac{\sigma}{d+\sigma} & \text { for } \alpha \geq \frac{1}{2}+\frac{\sigma}{2 d} \\ \delta=\frac{\sigma}{2 d} & \end{cases}
$$

Proof:

To check the abelian character of the algebra of fluctuation operators generated by $F^{\delta_{Q}}$ and $F^{\delta_{P}}$, it is enough to calculate the limit of commutator (cf. the definition of the symplectic form (1.16)):

$$
\lim _{\Lambda}\left[F_{\Lambda}^{\delta_{P}}, F_{\Lambda}^{\delta_{Q}}\right]=\lim _{\Lambda} \frac{1}{|\Lambda|^{1+\delta_{P}+\delta_{Q}}} \sum_{l, l^{\prime} \in \Lambda}\left[P_{l}, Q_{l^{\prime}}\right]=0
$$

The second part of the Proposition results from (2.27) and (2.28) which get on the critical line for $h=\hat{h} / V^{\alpha}$ the form:

$$
\lim _{\Lambda} \eta_{\Lambda}\left(\left\{\frac{1}{V^{\frac{1}{2}+\delta_{Q}}} \sum_{i \in \Lambda}\left(Q_{i}-\eta_{\Lambda}\left(Q_{i}\right)\right)\right\}^{2}\right)=\lim _{\Lambda} \frac{1}{V^{2 \delta_{Q}}} \frac{k T_{c}(\lambda)}{\Delta\left(c_{\Lambda, h}\left(T_{c}(\lambda), \lambda\right)\right)}
$$

and

$$
\lim _{\Lambda} \eta_{\Lambda}\left(\left\{\frac{1}{V^{\frac{1}{2}+\delta_{P}}} \sum_{i \in \Lambda}\left(P_{i}-\eta_{\Lambda}\left(P_{i}\right)\right)\right\}^{2}\right)=\lim _{\Lambda} \frac{1}{V^{2 \delta_{P}}} m k T_{c}(\lambda) .
$$

So the variance (3.22) is not trivial if and only if $\delta_{Q}=\gamma / 2$ and (4.18) is not trivial if and only if $\delta_{P}=0$.Hence, one gets $\delta_{Q}$ due to the lemma 3.1

Remark that if we put $\sigma=2$ in the preceding Proposition, then one gets the results for all short-range inhteractions $(\sigma \geq 2)$.

\section{C. $T_{c}\left(\lambda_{c}\right)=0$ : Quantum Fluctuations}

Lemma 3.3 If $(T, \lambda)$ coincides with the point $\left(0, \lambda_{c}\right)$, then the asymptotic volume behaviour of the gap $\Delta\left(c_{\Lambda, h}(0, \lambda), 0\right)$ is given by 


\section{HUGE INSTABILITIES DRIVEN BY QUANTUM FLUCTUATIONS}

$$
\gamma= \begin{cases}\text { if } d>\frac{3 \sigma}{2} & \text { for } \alpha<1=\alpha_{c} \\ \gamma=\frac{2}{3} \alpha & \text { for } \alpha \geq 1 \\ \gamma=\frac{2}{3} & \\ i f d=\frac{3 \sigma}{2} & \text { for } \alpha<1=\alpha_{c} \\ \gamma=\frac{2}{3} \alpha+0 & \text { for } \alpha \geq 1 \\ \gamma=\frac{1}{2}+0 & \\ i f \frac{\sigma}{2}<d<\frac{3 \sigma}{2} & \text { for } \alpha<\frac{1}{2}+\frac{3 \sigma}{4 d}=\alpha_{c} \\ \gamma=2 \alpha \frac{2 \sigma}{2 d+3 \sigma} & \text { for } \alpha \geq \frac{1}{2}+\frac{3 \sigma}{4 d} \\ \gamma=\frac{\sigma}{d} & \end{cases}
$$

Proof :

In that particular point of the critical line, the right side of Eq.(3.8) gets the following asymptotic form:

$$
O\left[\left(\frac{1}{V^{\alpha} \Delta}\right)^{2}+\frac{1}{V \Delta^{\frac{1}{2}}}\right] .
$$

As in the lemma 3.1 we define $\alpha_{c}$ such that $O\left[\left(1 / V^{\alpha_{c}} \Delta\right)^{2}\right]=O\left(1 / V \Delta^{\frac{1}{2}}\right)$ for three cases of the interaction decay:

$$
\begin{gathered}
\text { (a) } \frac{\sigma}{2}<d<\frac{3 \sigma}{2}, \\
\text { (b) } d=\frac{3 \sigma}{2}, \\
\text { (c) } d>\frac{3 \sigma}{2} .
\end{gathered}
$$

The conditions for $\alpha_{c}$ leads the asymptotic analysis of (3.8) to the case $\hat{h}=0$, so we again can profit by [8] where asymptotics are calculated for $h \equiv 0$

(a) $\frac{\sigma}{2}<d<\frac{3 \sigma}{2}$

From [8], we have for (3.24), when $\alpha=\alpha_{c}, O(\Delta)=$ $O\left(V^{\frac{\sigma}{d}}\right)$, when $\alpha=\alpha_{c}$. So we obtain straightfowardly

$$
\alpha_{c}=\frac{3 \sigma}{4 d}+\frac{1}{2}
$$

(b) $d=\frac{3 \sigma}{2}$.

Here, [8] yields $O(\Delta)=O\left(V^{-\left(\frac{2}{3}+0\right)}\right)$, so we obtain

$$
\alpha_{c}=1
$$

(c) $d>\frac{3 \sigma}{2}$.

In that case, [8] gives $O(\Delta)=O\left(V^{-\frac{2}{3}}\right)$, so

$$
\alpha_{c}=1 \text {. }
$$

If $\alpha \geq \alpha_{c}, \gamma$, then the last term in (3.24) dominates, hence $\gamma$ coincides with those for $\hat{h}=0$, or for $\alpha=\alpha_{c}$.
Now we pass to $\alpha<\alpha_{c}$ (a) $\frac{\sigma}{2}<d<\frac{3 \sigma}{2}$ Then the asymptotic form of Eq.(3.8) is

$$
\left[\left(\Delta+\Delta^{\frac{d}{\sigma}-\frac{1}{2}}+V^{\frac{\sigma}{2 d}-1}\right)\right]=\frac{1}{V^{2 \alpha} \Delta^{2}} .
$$

Then, straightforward calculations (just use $\frac{\sigma}{2}<d<\frac{3 \sigma}{2}$ ) yield

$$
\gamma=\alpha \frac{4 \sigma}{2 d+3 \sigma}
$$

(b) $d=\frac{3 \sigma}{2}$.

Then Eq.(3.8) transform to the following asymptotic form:

$$
O\left(\Delta+\Delta \ln (\Delta)+V^{-\frac{2}{3}}\right)=O\left(\frac{1}{V^{2 \alpha} \delta^{2}}\right)
$$

So we obtain

$$
\gamma=\frac{2 \alpha}{3}+0
$$

(c) $d>\frac{3 \sigma}{2}$

Now the asymptotic form of Eq.(3.8) becomes

$$
\left(\Delta+V^{\frac{\sigma}{2 d}-1}\right)=O\left(\frac{1}{V^{2 \alpha} \Delta^{2}}\right) .
$$

Due to the the condition $d>3 \sigma / 2$, we find

$$
\gamma=\frac{2 \alpha}{3}
$$

This complete the demonstration of the lemma

Proposition 3.4 If $(T, \lambda)$ coincides with the point $\left(0, \lambda_{c}(0)\right)$, then the algebra of fluctuation operators is non-abelian and the position fluctuation operator $F_{\delta_{Q}}(Q)$ is abnormal $\left(\delta_{Q}>0\right)$, while the momentum fluctuation operator $F_{\delta_{P}}(P)$ is supernormal with $\delta_{P}=-\delta_{Q}$ and

$$
\delta_{Q}= \begin{cases}\text { if } d>\frac{3 \sigma}{2} & \text { for } \alpha<1=\alpha_{c} \\ \delta=\frac{1}{6} \alpha & \text { for } \alpha<1=\alpha_{c} \\ d=\frac{3 \sigma}{2} & \text { for } \alpha \geq 1 \\ \delta=\frac{1}{6} \alpha+0 & \\ \delta=\frac{1}{6}+0 & \text { for } \alpha<\frac{1}{2}+\frac{3 \sigma}{4 d}=\alpha_{c} \\ i f \frac{\sigma}{2}<d<\frac{3 \sigma}{2} & \text { for } \alpha \geq \frac{1}{2}+\frac{3 \sigma}{4 d} \\ \delta=\alpha \frac{\sigma}{2 d+3 \sigma} & \\ \delta=\frac{\sigma}{4 d} & \end{cases}
$$

Proof:

If $\beta=(k T)^{-1} \rightarrow \infty$, variances (2.27) and (2.28) become 


\title{
V. A. ZAGREBNOV
}

$$
\lim _{\Lambda} \eta_{\Lambda}\left(\left\{\frac{1}{V^{\frac{1}{2}+\delta_{Q}}} \sum_{i \in \Lambda}\left(Q_{i}-\eta_{\Lambda}\left(Q_{i}\right)\right)\right\}^{2}\right)=\lim _{\Lambda} \frac{1}{V^{2 \delta_{Q}}} \frac{\lambda}{\sqrt{\Delta\left(c_{\Lambda, h}\left(T_{c}(\lambda), \lambda\right)\right)}}
$$

and

$$
\lim _{\Lambda} \eta_{\Lambda}\left(\left\{\frac{1}{V^{\frac{1}{2}+\delta_{P}}} \sum_{i \in \Lambda}\left(P_{i}-\eta_{\Lambda}\left(P_{i}\right)\right)\right\}^{2}\right)=\lim _{\Lambda} \frac{1}{V^{2 \delta_{P}}} \frac{\lambda m}{2} \sqrt{\Delta\left(c_{\Lambda, h}\left(T_{c}(\lambda), \lambda\right)\right)} .
$$

So one has just to apply the lemma 3.3 with $\delta_{Q}=$ $\gamma / 4=-\delta_{P}$ to get $\delta_{Q}$.

As far as $\delta_{Q}+\delta_{P}=0$, the non-abelian nature of the algebra of fluctuation operators is given by (1.6)

\section{CONCLUSION}

Results of the Sect.2 show that the structural phase transition driven by individual fluctuations of atoms strongly depends on their intensivity and the nature: large quantum fluctuations of light atoms can suppress the phase transition. On the other hand, statements of the Sects.3,4 demonstrate that in the case of existence of the critical line collective fluctuations show sensitivity on such perturbations as infinitisimal external fields. This kind of behaviour is typical for systems with developed quantum fluctuations such as, e.g., Bose systems in condensed phase,see [20].

\section{ACKNOWLEDGEMENTS}

Part of this paper forms the DEA-thesis of my student Patrice Joussot. I would like to thank him for efficient assistence made this publication possible.
[1] D. Ya. Petrina, Mathematical Foundations of Quantum Statistical Mechanics (Kluwer Academic Publishers, Dordrecht-London 1996).

[2] G. L. Sewell, Quantum Theory of Collective Phenomena (Claredon press, Oxford, 1986).

[3] D. Goderis, A. Verbeure, P. Vets, About the Mathematical Theory of Quantum Fluctuations in Leuven Notes in Mathematical and Theoretical Physics A 1, 31, (1989).

[4] D. Goderis, A. Verbeure, P. Vets, Commun. Math. Phys. 128, 533 (1990).

[5] D. Goderis, A. Verbeure, P. Vets, Prob. and Rel. Field 82, 527 (1987).

[6] D. Goderis, A. Verbeure, P. Vets, J. Stat. Phys. 62, 759 (1991)

[7] A. Verbeure, V. A. Zagrebnov, J. Stat. Phys. 69, 329 (1992).

[8] A. Car, V. A. Zagrebnov, Physica A 212, 398 (1994).

[9] A. Verbeure, V. A. Zagrebnov, J. Stat. Phys. 79, 377 (1995).

[10] A. Verbeure, V. A. Zagrebnov, Physica A 215, 394
(1995).

[11] N. N. Bogoliubov, Selected Works, (Naukova Dumka, Kiev, 1971).

[12] J. G. Brankov, V. A. Zagrebnov, N. S. Tonchev, Theor. Math. Phys. 66, 72 (1986).

[13] A. Patrick, J. Stat. Phys. 75, 253 (1994).

[14] M. Broidioi, B. Momont, A. Verbeure, J. Math. Phys. 36, 6746 (1995).

[15] A. D. Bruce, K. A. Cowley, Structural Phase Transition Taylor $\&$ Francis (London, 1981).

[16] V. L. Aksenov, N. M. Plakida, S. Stamenkovič, Neutron Scattering by Ferroelectrics (World Scientific, Singapore, 1990).

[17] S. Stamenkovič, N. S. Tonchev, V. A. Zagrebnov, Physica A 145, 262 (1987).

[18] K. A. Müller, W. Berlinger, E. Tossati, Z. Phys. B Condensed Matter 84, 277 (1991).

[19] A. Verbeure, V. A. Zagrebnov, J. Phys. A 28, 5415 (1995).

[20] I. A. Vakarchuk, Theor. Math. Phys. 65, 285 (1986).

\section{ВЕЛИКІ НЕСТІЙКОСТІ, СПРИЧИНЕНІ КВАНТОВИМИ ФЛЮКТУАЦЯМИ}

\author{
В. А. Загребнов \\ Центр теоретичної фізики і фізичний факультет Університету Медітеране (Екс-Марсель II), \\ Люміні-Каз 907, F-12388 Марсель, Седекс 09, Франиія \\ E-mail: zagrebnov@cpt.univ-mrs.fr
}

Пропонується квантовофлюктуаційний підхід як ключ до розуміння нестійкостей в квантовій сегнетоелектричній моделі. 